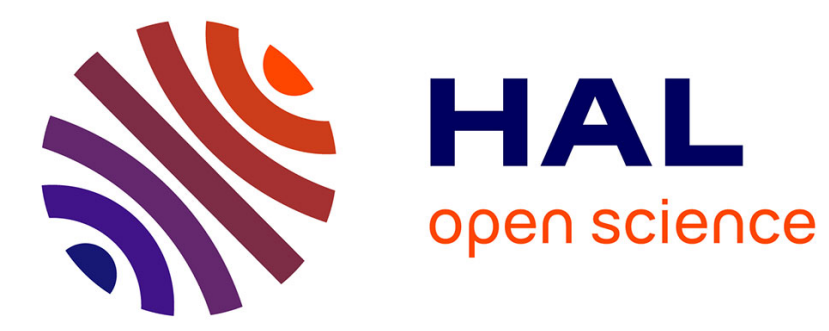

\title{
Novel Mesoporous Filters for Selective Gas Absorption
}

Marc Baumbach, Andreas Schütze, Fei Cheng, Steven Kelly, Hervé Delprat, Frédéric Parret, Philippe Menini, Aikaterini Soulantika, Bruno Chaudret, André Maisonnat

\section{- To cite this version:}

Marc Baumbach, Andreas Schütze, Fei Cheng, Steven Kelly, Hervé Delprat, et al.. Novel Mesoporous Filters for Selective Gas Absorption. IEEE Sensors, Oct 2004, Vienna, Austria. 10.1109/ICSENS.2004.1426326 . hal-02160584

\section{HAL Id: hal-02160584 https://hal.laas.fr/hal-02160584}

Submitted on 19 Jun 2019

HAL is a multi-disciplinary open access archive for the deposit and dissemination of scientific research documents, whether they are published or not. The documents may come from teaching and research institutions in France or abroad, or from public or private research centers.
L'archive ouverte pluridisciplinaire HAL, est destinée au dépôt et à la diffusion de documents scientifiques de niveau recherche, publiés ou non, émanant des établissements d'enseignement et de recherche français ou étrangers, des laboratoires publics ou privés. 


\title{
Novel Mesoporous Filters for Selective Gas Absorption
}

\author{
Marc Baumbach ${ }^{1}$; Andreas Schütze ${ }^{1}$; Fei Cheng ${ }^{2}$; Steven Kelly ${ }^{2}$; Hervé Delprat ${ }^{3}$; Frederic \\ Parret $^{4}$; Philippe Menini ${ }^{4}$; Katerina Soulantica ${ }^{5}$; Bruno Chaudret $^{5}$ and André Maisonnat ${ }^{5}$ \\ ${ }^{1}$ Saarland University, Lab for Measurement Technology, Saarbrücken, Germany, email m.baumbach@lmt.uni-saarland.de \\ ${ }^{2}$ University of Hull, Department of Physical Sciences, Hull, United Kingdom, email s.m.kelly@hull.ac.uk \\ ${ }^{3}$ MicroChemical Systems SA, Rue Porcena 15, 2035 Corcelles, Switzerland, email hdelprat@microchemical.com \\ ${ }^{4}$ Laboratoire d'Analyse et d'Architecture des Systèmes du CNRS, Toulouse, France email menini@laas.fr \\ ${ }^{5}$ Laboratoire de Chimie de Coordination du CNRS, Toulouse, France, email maisonat@lcc-toulouse.fr
}

\begin{abstract}
Semiconductor gas sensors have a proven potential for low cost and highly sensitive gas detection. However, selectivity remains one of the main problems for many applications. In this paper we report a new kind of mesoporous ceramic filter with nanoscale pores. The ceramic filter offers mechanical protection and can help to boost selectivity enomously through selective gas absorption. The developed nano-technological filters are based on mesoporous secondary and ternary silicon nitride derivatives with a defined particle size and are prepared via a new sol gel route. The interaction of the filters with $\mathrm{CO}, \mathrm{NO}_{2}, \mathrm{H}_{2}$ and propane were evaluated using broadband sensors. Highly selective absorption was observed for different gases, especially $\mathrm{NO}_{2}$. From these experiments a filter efficiency value is calculated which allows easy selection of suitable filter materials for different applications.
\end{abstract}

\section{Keywords}

Mesoporous filters, nanoparticle gas sensors, selective gas absorption.

\section{INTRODUCTION}

Semiconductor gas sensors offer high sensitivity, good reliability and low cost making them attractive for a wide range of applications ranging from air quality control to safety applications like explosion warning or fire detection. Current drawbacks are power consumption, especially for handheld devices, and also insufficient selectivity. In the frame of the EU project NANOSENSOFLEX two nanotechnological approaches are combined with advanced silicon microtechnology to achieve improved gas sensing systems based on miniaturized semiconductor gas sensors with sensitive layers based on novel doped and undoped metal oxide nanoparticles $[1,2]$. The achieved performance of the novel sensors which excels current commercial products was recently reported [3].

To improve the sensors further in terms of reproducibility, long term-stability and especially gas selectivity, filters based on materials exhibiting selective gas absorption are very attractive. For these filters mesoporous materials with nanoscale pores, which exhibit a large inner surface area, seem most suitable. The development and optimization of the synthetic protocols for the preparation of selective gas filters with a defined size and shape by a novel sol gel route to mesoporous secondary and ternary silicon nitride deriva-tives and their evaluation as selective gas filters was a second goal within the NANOSENSOFLEX project. Combined with advanced signal processing, this project aims at developing novel sensor configurations combining high miniaturization level and exceptional selectivity with a very low power consumption at low cost.

\section{EXPERIMENTAL}

\section{Filter material preparation}

Mesoporous silicon boron nitride was prepared as previously reported using a novel non-oxidic sol-gel procedure [4]. The synthesis of the transition metal containing silicon nitrides using a related procedure will be reported elsewhere [5]. The following table gives an overview of the filter material composition and the preparation route.

Table 1. Overview of material composition and preparation for five tested filter materials

\begin{tabular}{|l|l|}
\hline Number & Material composition and preparation \\
\hline FP1 & $\mathrm{Si}_{3} \mathrm{~N}_{4}-\mathrm{Pd}, 1000^{\circ} \mathrm{C}$ under $\mathrm{NH}_{3}$ flow \\
FP2 & $\begin{array}{l}\text { Silicon nitride membrane on } \alpha-\mathrm{Al}_{2} \mathrm{O}_{3} \\
\text { support (dipping for one time) }\end{array}$ \\
FP3 & $\mathrm{Si}_{3} \mathrm{~N}_{4}-\mathrm{BN}(\mathrm{Si}: \mathrm{B}=2: 3)$ \\
FP4 & $\mathrm{Si}_{3} \mathrm{~N}_{4}-\mathrm{Ni}, 1000^{\circ} \mathrm{C}$ under $\mathrm{NH}_{3}$ flow \\
FP5 & $\mathrm{Si}_{3} \mathrm{~N}_{4}-\mathrm{Pd}, 350^{\circ} \mathrm{C}$ under $\mathrm{H}_{2}$ flow \\
\hline
\end{tabular}

These filter materials exhibit a defined particle size and shape with nanoscale pores and a large surface area. After preparation, the mesoporous powder samples were isostatically pressed at 10 tons into a disk using the same process used for the preparation of a $\mathrm{KBr}$ disk for IR analysis. The nanosize pores of the ceramic filter material survived this process unchanged. The disks were then cut into $6 \mathrm{~mm}$ diameter filters using a cork borer to obtain the filter pellets used for the efficiency tests. 


\section{Hardware platform for test measurements}

A computer controlled gas test bench was used for the filter tests. The bench allows connection of up to six test gases injected into a carrier gas stream with adjustable relative humidity (r.h.). In these experiments synthetic air at 50\% r.h. with a flow rate of $500 \mathrm{ml} / \mathrm{min}$ was used. The test gases are injected into the carrier gas stream using three way valves to achieve almost instantaneous changes in the gas concentration. The data acquisition uses a multi-channel DMM (Keithley) for up to ten sensors in parallel.

For the filter evaluation a special filter chamber was designed into which the filter pellets are inserted. This filter chamber consists of several parts:

- housing for the filter pellet with male thread

- stamp and screw nut with female thread

- O-ring to center the filter-pellet in the housing and to avoid breaking the pellet when the stamp is screwed in

Rubber O-rings were used to achieve gas tight seals so that the gas can only pass the filter chamber through the filter pellet. Two sensor tests chambers for up to four sensors where installed, one in front of the filter chamber, another after the filter chamber to measure directly the difference in sensor response due to the filter pellet. The carrier gas stream passes through all three chambers. Before each experiment a leak test was performed to ensure that the gas passes through all three chambers. Fig. 1 shows the set-up for the filter tests. The total volume off the three chambers was approx. $10 \mathrm{ml}$, so that the gas concentration is changed in approx. 1,2 sec. These fast concentration changes allow determination of the sensor response time.

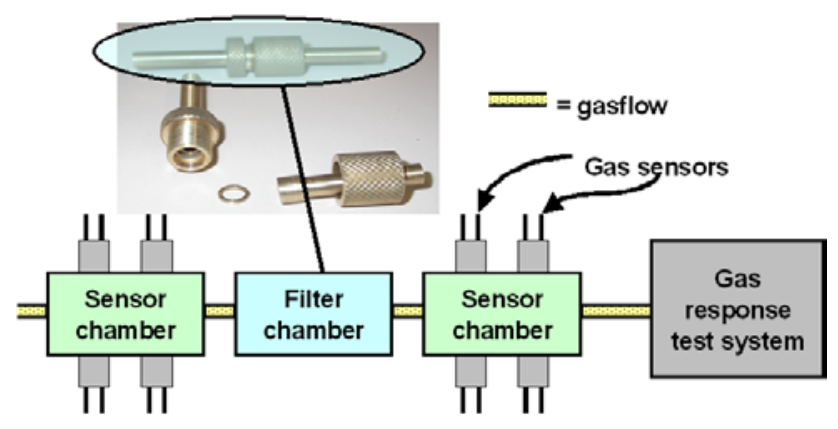

Note that the chosen set-up actually forces the gas under test through the filter. Effective removal of a given test gas under these conditions is therefore a better criterion for the filter efficiency than placing a filter pellet in front of the sensor element with the gas passing through by diffusion only. On the other hand, materials that prove effective under these test conditions should show an even better performance if gas transport in through diffusion only.

Four gases, $\mathrm{CO}, \mathrm{NO}_{2}, \mathrm{H}_{2}$ and propane $\left(\mathrm{C}_{3} \mathrm{H}_{8}\right)$, were chosen for the filter evaluation tests to cover a broad spectrum of common gases which might interfere with the target gas for a given application. We chose sensors with a broad detection spectrum to be able to evaluate all four gases using the same sensor. The sensors are microstructured Sisensors with $\mathrm{SnO}_{2}$ gas sensitive layer and noble metal doping mounted in standard TO-5 metal cans. These were screwed tightly into the sensor chambers. In all experiments, four sensors were placed in front of the filter and four behind to ensure correct operation of the gas test bench. The reported data compare the response of one sensor placed behind the filter chamber with the response of the same sensor in a reference measurement without filter so that a direct comparison of the response behavior is possible.

\section{RESULTS AND DISCUSSION}

For evaluation of the filter performance, the sensor response without filter is compared to the response with the different filter pellets placed in the filter chamber. The duration of the exposure was $20 \mathrm{~min}$ for each gas. Fig. 2 gives an overview of the sensor response to for all four gases and all five filter materials. It is immediately obvious that all filter materials except FP 1 are very effective for absorption of $\mathrm{NO}_{2}$, while FP 1 only slows the sensor reaction but ultimately leads to the same resistance change as the reference curve. After $\mathrm{NO}_{2}$ is switched off the sensor resistance remains higher than in the reference measurement and only slowly returns to the base line. Both effects can be explained with $\mathrm{NO}_{2}$ adsorbing on the filter material: when $\mathrm{NO}_{2}$ is injected in the carrier gas stream the full concentration reaches the sensor only after the filter is saturated. Then, when the gas is switched off again, $\mathrm{NO}_{2}$ is desorbing from the filter slowly so that the sensor is still exposed to a small $\mathrm{NO}_{2}$ concentration. Conversely, the response to $\mathrm{H}_{2}$ is nearly unaffected by all filters except FP 4 , which drastically reduces the sensor reaction.

To allow fast determination of the effectiveness of the different materials, we define the FE (filter efficiency) value as

$$
F E_{\text {red }}=1-\frac{\left(\frac{R_{0}-R_{\text {gas }}}{R_{0}}\right)_{\text {with Filter }}}{\left(\frac{R_{0}-R_{\text {gas }}}{R_{0}}\right)_{\text {without Filter }}}
$$

for reducing gases and

$$
F E_{o x}=1-\frac{\left(\frac{R_{g a s}-R_{0}}{R_{0}}\right)_{\text {with Filter }}}{\left(\frac{R_{g a s}-R_{0}}{R_{0}}\right)_{\text {without Filter }}}
$$

for oxidizing gases. With these definitions a FE-value of 1 denotes complete suppression of a given gas by the filter material while a value of 0 denotes no effect of the filter. 


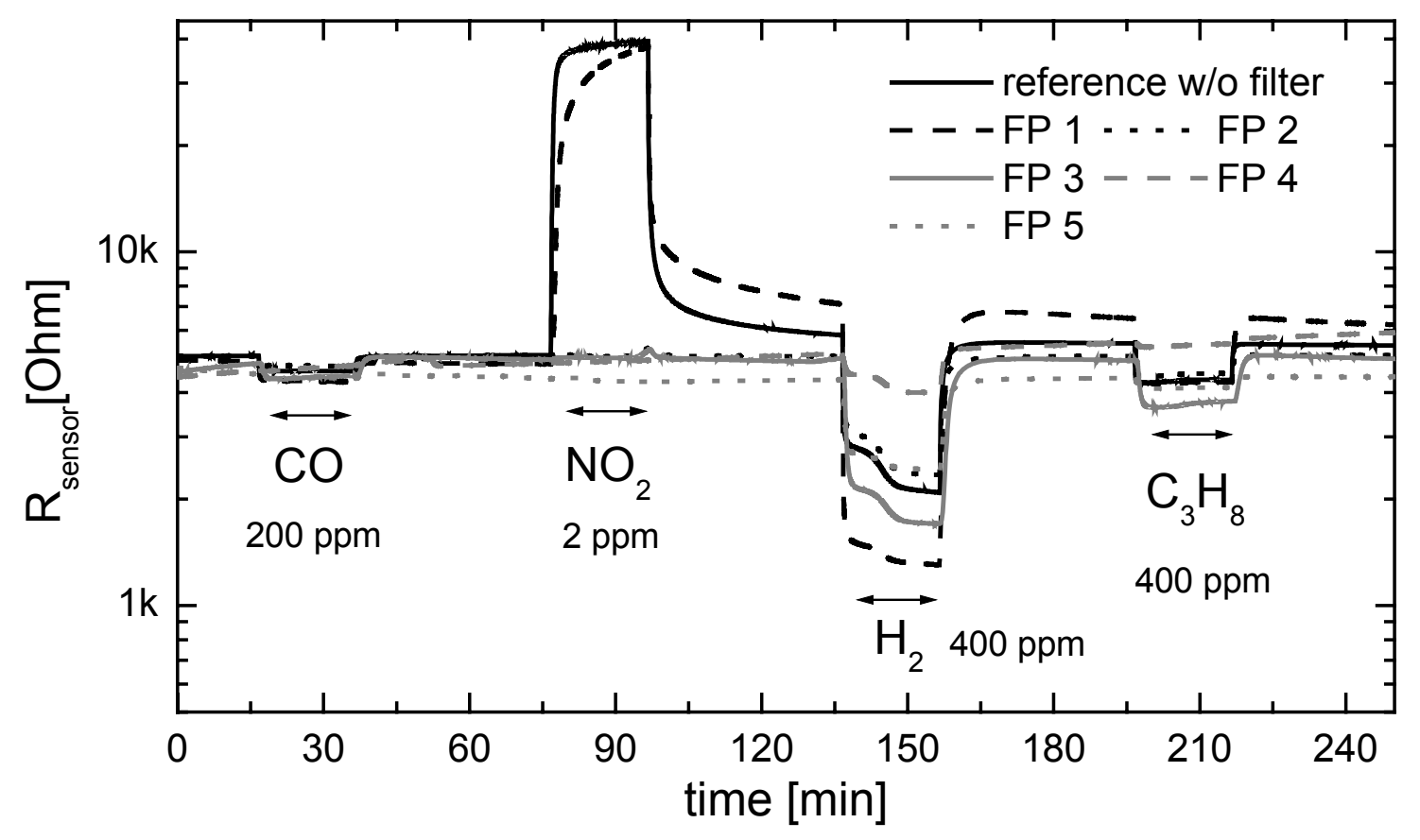

Fig. 3 gives an overview of the FE-values of all tested filter materials for all four test gases. With this plot, it is immediately obvious that FP 1 has no effect on the sensor response (although delaying the $\mathrm{NO}_{2}$ response as discussed above). The most promising materials seem to be FP 3, which removes $\mathrm{NO}_{2}$ very effectively without affecting the reaction to the other gases much, and FP 4, which effectively removes all gases except $\mathrm{H}_{2}$. The sensor response with these two filter materials is therefore shown in more detail in Fig. 4 in comparison with the reference curve without filter.

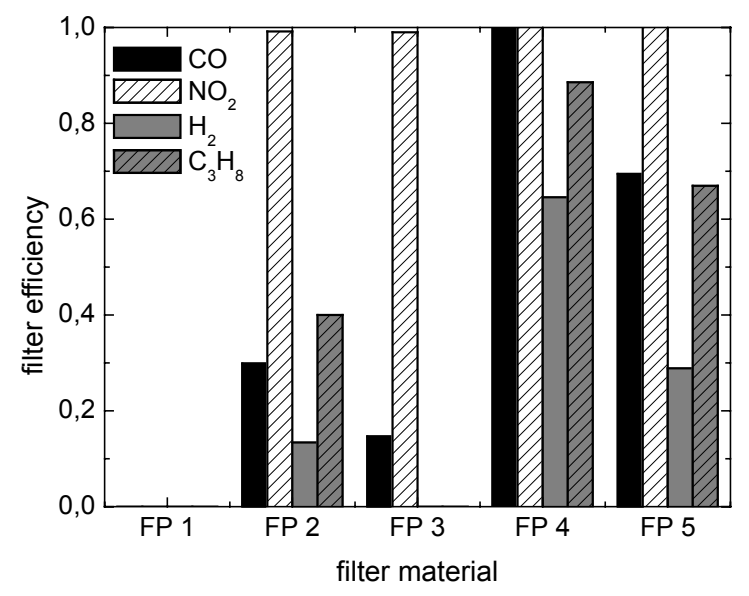

Figure 3. Filter efficiency for the five tested filter materials and the four test gases allowing fast assessment of the filter performance.

Both materials suppress the sensor response to $\mathrm{NO}_{2}$ completely so that the sensor resistance remains constant during the $20 \mathrm{~min}$ exposure. FP 3 leaves the sensor reaction to $\mathrm{CO}, \mathrm{H}_{2}$ and $\mathrm{C}_{3} \mathrm{H}_{8}$ practically unchanged. However, for all three gases a slight change in the response is evident: the raction is slower after the gas is switched on and the sensor resistance returns slowly to the baseline after the gas exposure. As for FP 1 and $\mathrm{NO}_{2}$ (see above) this can be explained with adsorption of gas molecules on the filter materials which then desorb slowly after the gas is switched off. FP 4, on the other hand, also suppresses the sensor response to $\mathrm{CO}$ and $\mathrm{C}_{3} \mathrm{H}_{8}$ almost completely and also reduces the response to $\mathrm{H}_{2}$ considerably. It is interesting to note that for this materials and the reaction to $\mathrm{H}_{2}$ the sensor signal return to the baseline is as fast as without filter. In this case, hydrogen obviously does not desorb from the filter material. This can either mean that the adsorption energy is too high for desorption at room temperature or that the filter catalyses a reaction between hydrogen and oxygen.

Of course, it is interesting to see if the sensor performance is unchanged for high gas concentrations, especially for $\mathrm{NO}_{2}$ where the tests were performed with a concentration of only $2 \mathrm{ppm}$, and for longer exposures. We checked this by increasing the $\mathrm{NO}_{2}$ concentration to $20 \mathrm{ppm}$ for an exposure of two hours. For this experiment, an undoped $\mathrm{SnO}_{2}$ sensor with extremely high $\mathrm{NO}_{2}$ sensitivity was chosen. The results for FP 4 are shown in Fig. 5. As can be seen, the sensor response is not completely suppressed as for the smaller concentration but still the resistance 


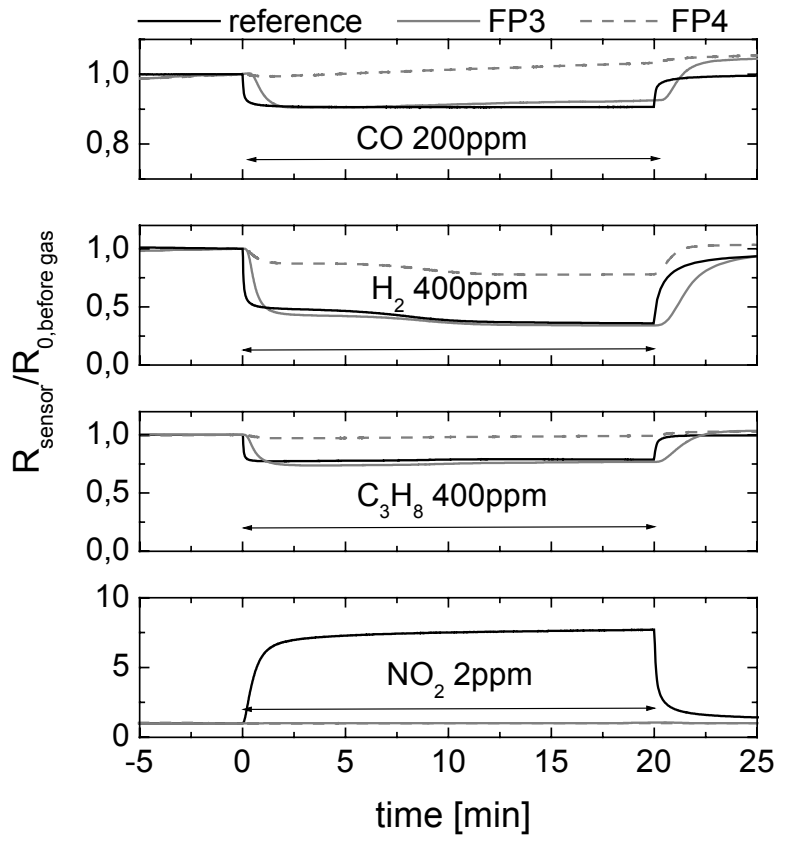

increase is reduced by a factor of approx. 5. Note that in this case, the initial response time with the filter material is shorter than without filter. This is due to a faster response time of the sensor at low $\mathrm{NO}_{2}$ concentrations. Note that even for the long exposure no filter breakthrough is evident. The slight increase during the exposure is the same with and without filter so this is probably not due to a breakdown of the filter but to a slow drift off the sensor during long exposure.

\section{CONCLUSIONS AND OUTLOOK}

These first tests have proven that the novel mesoporous filter materials offer considerable potential to improve the selectivity of gas sensor systems. With the materials studied so far, suppression of $\mathrm{NO}_{2}$ interference seems possible, especially for low concentrations. Also for hydrogen detection, FP 4 seems a promising material as it suppresses many different gases.

Further tests are required to investigate the mechanism of the filter performance: thermal desorption experiments will show if the filter function is due to an adsorption/desorption process or if catalytic effects play a role at least for some gases. In addition, tests need to be made with filter pellets placed in the sensor housing to check the filter performance if the gas is not pressed through the filter but is instead transported to the sensor by diffusion.
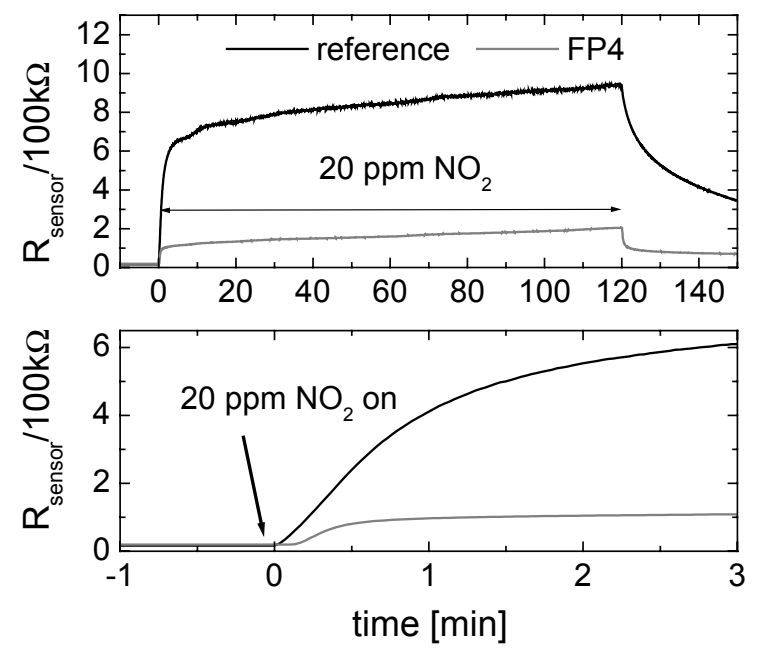

\section{ACKNOWLEDGMENTS}

Financial support of the European Commission within the program GROWTH under grant G5RD-CT-2002-00722 is gratefully acknowledged.

\section{REFERENCES}

[1] P. Fau, M. Sauvan, S. Trautweiler, C. Nayral, L. Erades, A. Maisonnat, and B. Chaudret, Sens. \& Actuators B 2001, 78, p. 83.

[2] P. Menini, F. Parret, M. Guerrero, A. Martinez, K. Soulantica, L. Erades, A. Maisonnat, and B. Chaudret, Sens. \& Actuators B, in press.

[3] A. Maisonnat, H. Delprat, M. Baumbach, A. Schütze, F. Cheng, S. Kelly, F. Parret, P. Menini, K. Soulantica, and B. Chaudret: "New Generation of Micro Machined Silicon Gas Sensors: Nano-structured Pd- and Ptdoped Tin Dioxide Gas Sensitive Layers for the Detection of Hazardous Gases", EUROSENSORS XVIII, Rome, Sep. 12-15, 2004.

[4] F. Cheng, B. Toury, F. Levebvre and J. S. Bradley, "Preparation of a mesoporous silicon boron nitride via a non-aqueous sol-gel route", Che. Commun., 242, 2003.

[5] F. Cheng, S. M. Kelly, S. Clark, M. G. Francesconi, N. A. Young, A. W. Jackson and J. S. Bradley, "Preparation of a mesoporous silicon nickel imidebromide complex and its pyrolytic conversion into a $\mathrm{Ni}-\mathrm{Si}_{3} \mathrm{~N}_{4}$ composite", Chem. Commun., to be submitted. 
